\title{
Deformation Correction in Brain MRI Using Mutual Information and Genetic Algorithm
}

\author{
Pradipta Maji B. Chanda M. K. Kundu \\ Center for Soft Computing Research \\ Indian Statistical Institute, Kolkata, India \\ \{pmaji,chanda,malay\}@isical.ac.in
}

\author{
Suman Dasgupta \\ Advanced Medicare and Research Institute \\ Salt Lake, Kolkata, West Bengal, India
}

\begin{abstract}
During MR imaging, the image of the object may get deformed due to the slope between the MR probe and the object. It is in general undesirable, particularly for $3 D$ imaging. In this paper we propose a novel method to reconstruct the undeformed image of the object by measuring the deformation at the time of the MR scan. The image registration technique coupled with genetic algorithm is used to predict the deformation of the object. The inverse of this deformation is then applied to the image, generating the image that would have been seen had there been no slope with the probe. The proposed algorithm has been tested on a set of brain MR images and shown to remove the deformation and so give improved $3 D$ reconstructions.
\end{abstract}

\section{Introduction}

In medical imaging technology, a number of complementary diagnostic tools such as x-ray computer tomography $(\mathrm{CT})$, magnetic resonance imaging (MRI) and position emission tomography (PET) are available. Magnetic resonance imaging (MRI) is an important diagnostic imaging technique for the early detection of abnormal changes in tissues and organs. Its unique advantage over other modalities is that it can provide multispectral images of tissues with a variety of contrasts based on the three MR parameters $\rho$, T1, and $\mathrm{T} 2$. Therefore, majority of research in medical image concerns MR images [5].

During MR imaging, the image of the object may get deformed due to inclination of the object. It is undesirable particularly for 3D imaging. Even though the inclination angle is small, it is sufficient to deform the object. It changes the detailed geometry of the image and the characteristic appearance of a lesion on which the diagnosis is based.

In this paper we propose a novel method which aims to reconstruct the undeformed shape of the object; that is, the scan which would have been achieved if there had been no inclination between object and probe. The basic postulates of the proposed method is that in the undeformed shape of the object the left- and right-hand sides of the object about the axis of symmetry will be geometrically aligned. That is, the mutual information between the left- and right-hand sides of the object about the axis of symmetry is maximum. The genetic algorithm has been used to predict the deformation due to inclination of object considering mutual information as the fitness function. The inverse of this deformation is then applied to the image, generating the undeformed image that would have been seen had there been no inclination problem. The proposed method has been tested on a set of brain MR images and shown to correct the deformation due to inclination problem and give improved $3 \mathrm{D}$ reconstructions.

The rest of the paper is organized as follows. In Section 2 some basic concepts about mutual information is introduced. The proposed algorithm is presented in Section 3. Section 4 presents the estimation of deformation parameters by using genetic algorithm. Experimental results are presented in Section 5. Finally, concluding remarks are given in Section 6.

\section{Mutual Information and Image Registration}

In this section, the basic concepts of mutual information and registration using mutual information are reported.

\subsection{Mutual Information}

Two discrete random variables $A$ and $B$ with marginal probability distributions $p_{A}(a)$ and $p_{B}(b)$ and joint probability distribution $p_{A B}(a, b)$ are statistically independent if $p_{A B}(a, b)=p_{A}(a) \cdot p_{B}(b)$; while they are maximally dependent if they are related by one-to-one mapping $T$ : $p_{A}(a)=p_{B}(T(a))=p_{A B}(a, T(a))$. The mutual information $I(A, B)$ of $A$ and $B$ measures the degree of depen- 
dence of $A$ and $B$ as the distance between the joint distribution $p_{A B}(a, b)$ and the distribution associated to the case of complete independence $p_{A}(a) \cdot p_{B}(b)$, by means of the Kullback-Leibler measure [6], that is,

$$
I(A, B)=\sum_{a, b} p_{A B}(a, b) \log \frac{p_{A B}(a, b)}{p_{A}(a) \cdot p_{B}(b)}
$$

Mutual information is related to the information theoretic notion of entropy by the following equations:

$$
\begin{aligned}
I(A, B)=H(A) & +H(B)-H(A, B) \\
& =H(A)-H(A \mid B) \\
& =H(B)-H(B \mid A)
\end{aligned}
$$

with $H(A)$ and $H(B)$ being the entropy of $A$ and $B$ respectively. $H(A, B)$ is their joint entropy, and $H(A \mid B)$ and $H(B \mid A)$ are the conditional entropy of $A$ given $B$ and of $B$ given $A$, respectively. $H(A), H(A \mid B)$ and $H(A, B)$ are defined as

$$
\begin{gathered}
H(A)=-\sum_{a} p_{A}(a) \log p_{A}(a) \\
H(A \mid B)=-\sum_{a, b} p_{A B}(a, b) \log p_{A \mid B=b}(a) \\
H(A, B)=-\sum_{a, b} p_{A B}(a, b) \log p_{A B}(a, b)
\end{gathered}
$$

with $p_{A \mid B=b}(a)$ the conditional probability of $A$ given $B=$ $b$. The entropy $H(A)$ is known to be a measure of the amount of uncertainty about the random variable $A$, while $H(A \mid B)$ is the amount of uncertainty left in $A$ when knowing $B$. Hence, from $3, I(A, B)$ is the reduction in the uncertainty of the random variable $A$ by the knowledge of another random variable $B$, or, equivalently, the amount of information that $B$ contains about $A$. If $A$ and $B$ are independent, $p_{A B}(a, b)=p_{A}(a) \cdot p_{B}(b)$ and $I(A, B)=0$; while if $A$ and $B$ are one-to-one related, $I(A, B)=H(A)=H(B)$. Some properties of mutual information are reported next.

\begin{tabular}{|ll|}
\hline Independence : & $I(A, B)=0$ \\
Symmetry : & $I(A, B)=I(B, A)$ \\
Self information : & $I(A, A)=H(A)$ \\
Lower bound : & $I(A, B) \geq 0$ \\
Upper bound : & $I(A, B) \leq \min (H(A), H(B))$ \\
& $\leq(H(A)+H(B)) / 2$ \\
& $\leq \max (H(A), H(B))$ \\
& $\leq H(A, B)$ \\
& $\leq H(A)+H(B)$ \\
\hline
\end{tabular}

\subsection{Mutual Information as Registration Criterion}

If $\mathcal{A}$ and $\mathcal{B}$ are two images that are geometrically related by the registration transformation $T_{\alpha}$ with parameters $\alpha$ such that pixel $\mathbf{p}$ in $\mathcal{A}$ with intensity a physically corresponds to pixel $T_{\alpha}$ in $\mathcal{B}$ with intensity $b$, the statistical dependence between $a$ and $b$ or the information that one value contains about the other is measured by the mutual information $I(A, B)$ of the variables $A=\{a\}$ and $B=\{b\}$

$$
\begin{gathered}
a=\mathcal{A}(p) \\
b=\mathcal{B}\left(T_{\alpha}(p)\right) \\
I(A, B)=\sum_{a, b} p_{A B}(a, b) \log \frac{p_{A B}(a, b)}{p_{A}(a) \cdot p_{B}(b)}
\end{gathered}
$$

with $p_{A B}(a, b), p_{A}(a)$ and $p_{B}(b)$ the joint and marginal distributions of the pair $(a, b)$ and of $a$ and $b$, respectively $[1,3]$. Estimates for these distributions can be obtained by simple normalization of the joint and marginal histograms of the overlapping parts of both images. The relationship $p_{A B}(a, b)$ between $a$ and $b$, and hence, their mutual information $I(A, B)$ depends on $T_{\alpha}$, that is, on the registration of the images. The mutual information registration criterion postulates that the images are geometrically aligned by the transformation $T_{\alpha^{\star}}$ for which $I(A, B)$ is maximal [7]

$$
\alpha^{\star}=\arg \max _{\alpha} I(A, B)
$$

\section{Proposed Algorithm}

In this section, we propose a method to remove perspective abnormalities from an image using image registration in a single image. The proposed method consists of three phases:

1. Determination of axis of symmetry;

2. Rotation of image about centroid; and

3. Image modification for improved definition.

Each of the three methods is elaborated next one by one.

\subsection{Determination of Axis of Symmetry}

Let $F=[f(x, y)]$ be an image of size $M \times N$ where $f(x, y)$ is the gray value at location $(x, y)$ in $F, x=$ $0,1,2, \cdots, M-1$ and $y=0,1,2, \cdots, N-1$. Considering gray level as point mass, $F$ is considered as composed of a set of point masses located at the points $(x, y)$. Then, the $(i, j)$ th moment of $F$ with respect to origin is defined by

$$
m_{i j}=\sum_{x} \sum_{y} x^{i} y^{j} f(x, y)
$$

$m_{00}$ is the total mass of $F$ and $m_{02}$ and $m_{20}$ are the moments of inertia of $F$ around $x$ and $y$ axis respectively. The centroid of $F$ is the point $(\bar{x}, \bar{y})$ defined by

$$
\bar{x}=\frac{m_{10}}{m_{00}} ; \quad \bar{y}=\frac{m_{01}}{m_{00}}
$$


Considering the origin at the centroid, moments computed with respect to this origin are called central moments and be denoted by $\bar{m}_{i j}$. Thus,

$$
\bar{m}_{00}=m_{00} ; \quad \bar{m}_{01}=\bar{m}_{10}=0
$$

The moment of inertia of $F$ about the line $\left(y-y_{0}\right) \cos \theta=(x-$ $\left.x_{0}\right) \sin \theta$, which is the line through $\left(x_{0}, y_{0}\right)$ with slope $\theta$ is

$$
\sum \sum\left[\left(x-x_{0}\right) \sin \theta-\left(y-y_{0}\right) \cos \theta\right]^{2} f(x, y)
$$

The moment of inertia around the minimum-inertia line must be

$$
\sum \sum[(x-\bar{x}) \sin \theta-(y-\bar{y}) \cos \theta]^{2} f(x, y)
$$

which is the moment of inertia about the line of slope $\theta$ through $(\bar{x}, \bar{y})$. That is, the minimum-inertia line passes through the centroid of $F$. This line is called the principal axis of $F$ or the axis of symmetry of $F$. The slope of the principal axis of $F$ is given by [4]

$$
\theta=\frac{1}{2} \tan ^{-1} \frac{2 \bar{m}_{11}}{\bar{m}_{20}-\bar{m}_{02}}
$$

\subsection{Rotation of Image about Centroid}

Let $\dot{F}^{\prime}=\left[\dot{f}\left(\dot{x}, \dot{y}^{\prime}\right)\right]$ be the rotated image after rotation of $F$ about the centroid $(\bar{x}, \bar{y})$ with angle $-\theta$. The new coordinates $\left(\dot{x}, \dot{y}^{\prime}\right)$ of $\dot{F}$ correspond to the old coordinates $(x, y)$ are as follows

$$
\begin{gathered}
x=(\dot{x}-\bar{x}) \cos \theta+(\dot{y}-\bar{y}) \sin \theta+\bar{x} \\
y=-(\dot{x}-\bar{x}) \sin \theta+(\dot{y}-\bar{y}) \cos \theta+\bar{y}
\end{gathered}
$$

A gray level is assigned to the new coordinates $(x, y)$ by interpolating between the gray levels of the original image $F$ at the points surround $(x, y)$. Let $x_{1}=\lfloor x\rfloor$ and $y_{1}=\lfloor y\rfloor$. As per bilinear interpolation, the gray level that is assigned to $\left(x^{\prime}, y^{\prime}\right)$ is given by

$$
\begin{array}{r}
f(\dot{x}, \hat{y})=\left(1-w_{x}\right)\left(1-w_{y}\right) f\left(x_{1}, y_{1}\right)+\left(1-w_{x}\right) w_{y} \\
f\left(x_{1}, y_{1}+1\right)+w_{x}\left(1-w_{y}\right) f\left(x_{1}+1, y_{1}\right)+ \\
w_{x} w_{y} f\left(x_{1}+1, y_{1}+1\right)
\end{array}
$$

where $w_{x}=x-x_{1}$ and $w_{y}=y-y_{1}$ respectively.

\subsection{Image Modification for Improved Definition}

Rotating the image $F$ about the centroid $(\bar{x}, \bar{y})$ with angle $\theta$, the rotated image $F^{\prime}$ is modified accordingly. Let $\tilde{F}=[\tilde{f}(\tilde{x}, \tilde{y})]$ be the modified image after expansion of image $\dot{F}^{\prime}=[\dot{f}(\dot{x}, \dot{y})]$. Let the left and right hand portions of the image $F^{\prime}$ is expanded by $\delta$ and $\frac{1}{\delta}$ about the axis of symmetry respectively. For right hand portion, the new coordinates $(\tilde{x}, \tilde{y})$ of $\tilde{F}$ correspond to the old coordinates $(\dot{x}, y)$ as follows

$$
\dot{x}=\tilde{x} ; \quad \underline{y}=\bar{y}+\frac{(\tilde{y}-\bar{y})}{\delta}
$$

Similarly, for left hand portion, the new coordinates $(\tilde{x}, \tilde{y})$ of $\tilde{F}$ correspond to the old coordinates $(x, y)$ as follows

$$
\dot{x}=\tilde{x} ; \quad \dot{y}=\bar{y}-\delta(\bar{y}-\tilde{y})
$$

A gray level is assigned to the new coordinates $(\tilde{x}, \tilde{y})$ by interpolating between gray levels of the image $F$ at the points surround $(x, y)$. Let $y_{2}=\lfloor y\rfloor$. For right hand portion, the gray value that is assigned to $(\tilde{x}, \tilde{y})$ is given by

$$
\tilde{f}(\tilde{x}, \tilde{y})=\left(1-w_{y}\right) f^{\prime}\left(\dot{x}, y_{2}\right)+w_{y} \dot{f}\left(\dot{x}, y_{2}+1\right)
$$

while for left hand portion, the gray value that is assigned to $(\tilde{x}, \tilde{y})$ is given by

$$
\tilde{f}(\tilde{x}, \tilde{y})=\left(1-w_{y}\right) \dot{f}\left(\dot{x}, y_{2}\right)+w_{y} \dot{f}\left(\dot{x}, y_{2}-1\right)
$$

where $w_{y}=\dot{y}-y_{2}$.

\subsection{Image Registration}

To evaluate the quality of modified image $\tilde{F}$, one portion about the axis of symmetry of $\tilde{F}$ is registered in the other portion of $\tilde{F}$. Let the left hand portion of $\tilde{F}$ is selected to be the floating image $\mathcal{F}$ from which samples are taken and registered into the right hand portion which is considered as reference image $\mathcal{R}$.

Let $p$ and $q$ denote the image intensities in the floating image $\mathcal{F}$ and reference image $\mathcal{R}$ at position $(x, y)$ from the axis of symmetry respectively. The joint image intensity histogram $h(p, q)$ of both images is computed by binning the image intensities pairs $(p, q)$. The total number of bins in the joint histogram is $\left(x_{\max }-x_{\min }+1\right) \times\left(x_{\max }-x_{\min }+1\right)$ where $x_{\text {max }}$ and $x_{\text {min }}$ be the maximum and minimum gray levels of the given image. Estimations for the marginal and joint image intensity distributions $P(p), P(q)$, and $P(p, q)$ are obtained by normalization of $h(p, q)$

$$
\begin{aligned}
P(p, q) & =\frac{h(p, q)}{\sum_{p, q} h(p, q)} \\
P(p) & =\sum_{q} P(p, q) \\
P(q) & =\sum_{p} P(p, q)
\end{aligned}
$$

The mutual information registration criterion $I(\alpha)$ is then evaluated by

$$
I(\alpha)=\sum_{p, q} P(p, q) \log _{2} \frac{P(p, q)}{P(p) \cdot P(q)}
$$


and the optimal registration parameter $\alpha^{\star}$ is found from

$$
\alpha^{\star}=\arg \max _{\alpha} I(\alpha)
$$

\section{Genetic Algorithm Evolution}

The basic structure of genetic algorithm (GA) [2] revolves around the concept of evolving successive solutions according to their fitness. The fitness function is elaborated next.

\subsection{Fitness Function}

The fitness of each solution is determined using the mutual information registration criterion defined earlier.

For the purpose of evolution, each solution has to be encoded in bit string format (chromosome). Three major functions - selection, crossover and mutation, as developed in the current GA formulation, are discussed next.

\subsection{Chromosome}

In the proposed method, a chromosome is a binary string of length 25 consisting of three parts:

1. change in centroid $(\Delta x, \Delta y)$;

2. change in slope of principal axis $(\Delta \theta)$; and

3. change in $\delta(\Delta \delta)$.

The 1st, 5th, 9th, and 17th positions of the chromosome represent the sign bits of $\Delta x, \Delta y, \Delta \theta, \Delta \delta$ respectively. The values of $\Delta x$ and $\Delta y$ are represented by 3-bit binary strings while $\Delta \theta$ and $\Delta \delta$ are represented by 7-bit and 8-bit binary strings respectively. The 3-bit binary strings corresponding to $\Delta x$ and $\Delta y$ are converted into their decimal equivalents; while the 7- and 8-bit binary strings corresponding to $\Delta \theta$ and $\Delta \delta$ are first converted into their decimal equivalents and then divided by $2^{7} \times 10^{3}$ and $10^{3}$ respectively. Hence,

$$
\begin{gathered}
0 \leq \Delta x \leq 7 ; \quad 0 \leq \Delta y \leq 7 \\
0.000007 \leq \Delta \theta \leq 0.001 ; \quad 0.000 \leq \Delta \delta \leq 0.255
\end{gathered}
$$

\subsection{Selection}

Selection is done by the roulette wheel method. The probabilities are calculated on the basis of ranking of the individuals in terms of the fitness function, instead of the fitness function itself. Elitism is incorporated in the selection process to prevent oscillation of the fitness function with generation. The fitness of the best individual of a new generation is compared with that of the current generation. If the later has a higher value the corresponding individual replaces a randomly selected individual in new population.

\subsection{Crossover Algorithm}

The crossover algorithm implemented here is similar in nature to the conventional one normally used for GA framework. It takes two chromosomes from the present population (PP) and forms the resultant chromosome. Like a single point crossover, it sets a crossover point and each half about the crossover point is selected from the two respective chromosomes to form the offsprings.

\subsection{Mutation Algorithm}

The mutation algorithm emulates the normal mutation scheme. It makes some minimal change in the existing chromosome of PP (present population) to form a new chromosome for NP (next population). Similar to conventional single point mutation, the chromosome is mutated at a single point.

The experimental results reported next confirm that the GA evolution provides the desired direction to arrive at the best solution to correct the structural deformation of brain MR images.

\section{Implementations and Experimental Results}

This section presents the application of the proposed method to reconstruct the undeformed image of the object. The major metric for evaluating the interpolation method used in the proposed method is peak-signal-to-noise-ratio (PSNR). The PSNR in decibels $(\mathrm{dB})$ is computed by using

$$
\text { PSNR }=20 \log _{10} \frac{(\text { Level }-1)}{\text { RMSE }}
$$

where RMSE (root mean squared error) is the square root of MSE (mean squared error), which is given by

$$
\mathrm{MSE}=\frac{\sum_{x=1}^{M} \sum_{y=1}^{N}\left(f(x, y)-f_{1}(x, y)\right)^{2}}{M \times N}
$$

where $F=[f(x, y)]$ is the original image; and $F_{1}=$ $\left[f_{1}(x, y)\right]$ is the reconstructed image obtained from $\tilde{F}=$ $[\tilde{f}(\tilde{x}, \tilde{y})]$ using deformation parameters. We also report position of centroid $(\bar{x}, \bar{y})$, slope $(\theta)$, and expansion coefficient $(\delta)$. The GA parameters used here are as follows:

\begin{tabular}{|c|c|}
\hline Maximum generation : & 100 \\
Population size : & 100 \\
Chromosome length : & 25 \\
Probability of crossover : & 0.8 \\
Probability of mutation : & 0.01 \\
\hline
\end{tabular}

The parameters are held constant across all runs. Unbiased initial population is generated randomly spreading over entire variable space in consideration. More than 100 brain 
Table 1. Deformation Parameters and PSNR

\begin{tabular}{|c|c|c|c|c|c|c|}
\hline No & Slice & $M \times N$ & $(\bar{x}, \bar{y})$ & $\theta$ & $\delta$ & PSNR \\
\hline 1 & 1 & 256,256 & 129,131 & 4.91 & 1.12 & 49.6 \\
1 & 2 & 256,256 & 128,131 & 4.90 & 1.12 & 51.2 \\
1 & 3 & 256,256 & 130,129 & 4.78 & 1.12 & 47.1 \\
\hline 2 & 1 & 512,360 & 254,177 & 5.01 & 1.11 & 46.3 \\
2 & 2 & 512,360 & 255,181 & 5.21 & 1.12 & 50.0 \\
2 & 3 & 512,360 & 254,179 & 5.01 & 1.12 & 49.1 \\
\hline 3 & 1 & 512,512 & 255,249 & 4.11 & 1.13 & 44.2 \\
3 & 2 & 512,512 & 257,253 & 4.19 & 1.12 & 47.3 \\
3 & 3 & 512,512 & 255,251 & 4.04 & 1.12 & 48.1 \\
\hline
\end{tabular}
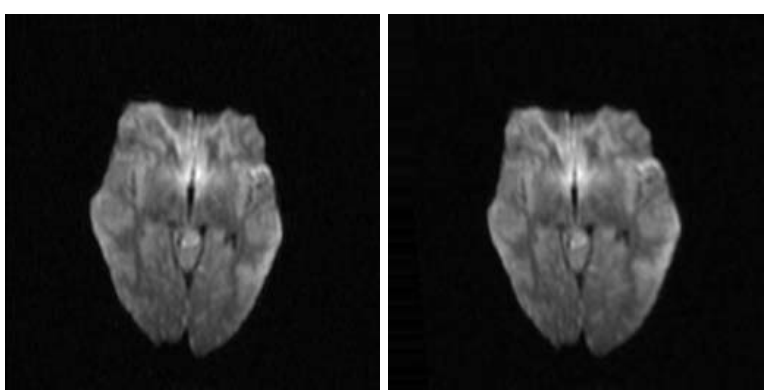

Figure 1. Deformed and corrected images

MRI with different size and 16 bit gray levels are tested. The proposed method is implemented in $\mathrm{C}$ language and compiled in LINUX environment having machine configuration Pentium IV, $3.2 \mathrm{GHz}, 1 \mathrm{MB}$ cache, and 1 GB RAM.

Fig.1-3 represent the input deformed images and their corresponding corrected images obtained through the proposed method. Table 1 represents the values of deformation parameters obtained using GA evolution and the PSNR value. While first to third columns represent the sequence number, slice number, and slice size of the 3D image of the object, fourth to six columns depict the values of centroid, slope, and magnification coefficient derived through GA run. Seventh column shows the PSNR value. All the results reported in Table 1 establish the facts that:

1. the expansion coefficient $\delta$ is constant for all the slices of all the sequences of a given 3D image;

2. the position of centroid $(\bar{x}, \bar{y})$ and slope $\theta$ remain same for all the slices of a particular sequence; and

3. the high PSNR values of different slices establish the high quality of the interpolation method used in the proposed scheme.
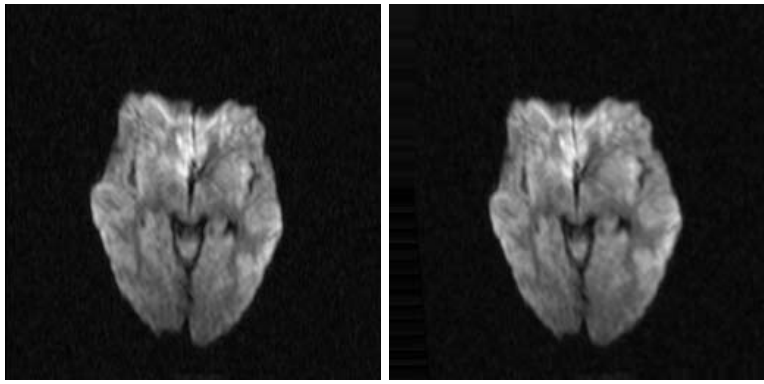

Figure 2. Deformed and corrected images
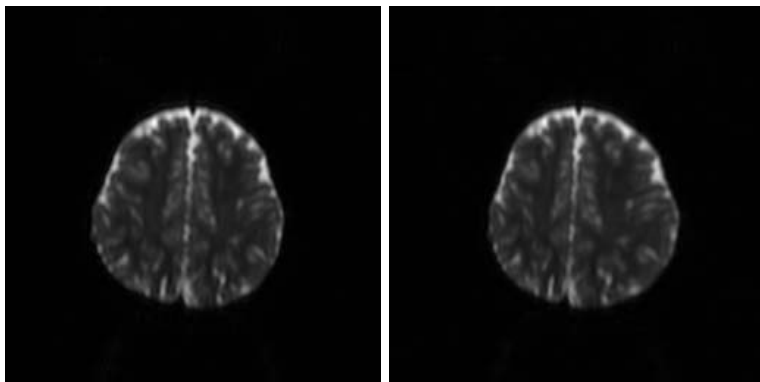

Figure 3. Deformed and corrected images

\section{Conclusion}

This paper presents a novel method to correct the deformation of brain MR images due to inclination of the object. The genetic algorithm coupled with mutual information is used to predict the deformation. Extensive experimental results on a set of brain MR images establish that the proposed method has been shown to remove the deformation and give improved 3D reconstructions.

\section{References}

[1] L. G. Brown. A Survey of Image Registration Techniques. ACM Computing Surveys, 24(4):325-376, December 1992.

[2] D. E. Goldberg. Genetic Algorithms in Search, Optimizations and Machine Learning. Morgan Kaufmann, 1989.

[3] C. R. Maurer and J. M. Fitzpatrick. A Review of Medical Image Registration. in Interactive Image-Guided Neurosurgery, R. J. Maciunas, Ed. Park Ridge, IL: Amer. Association of Neurological Surgeons, pages 17-44, 1993.

[4] A. Rosenfeld and A. C. Kak. Digital Picture Processing, chapter 9. Academic Press, Inc., 1982.

[5] P. Suetens. Fundamentals of Medical Imaging, chapter 6. Cambridge University Press, 2002.

[6] I. Vajda. Theory of Statistical Inference and Information. Dordrecht, The Netherlands: Kluwer, 1989.

[7] I. W. M. Wells, P. Viola, H. Atsumi, S. Nakajima, and R. Kikinis. Multi-modal Volume Registration by Maximization of Mutual Information. Med. Image Anal., 1(1):35-51, March 1996. 